IFUP-TH 6/94

January 1994

gr-qc/9401027

\title{
Black Holes as Quantum Membranes
}

\author{
Michele Maggiore \\ I.N.F.N. and Dipartimento di Fisica dell'Università, \\ piazza Torricelli 2, I-56100 Pisa, Italy.
}

\begin{abstract}
We propose a quantum description of black holes. The degrees of freedom to be quantized are identified with the microscopic degrees of freedom of the horizon, and their dynamics is governed by the action of the relatistic bosonic membrane in $D=4$. We find that a consistent and plausible description emerges, both at the classical and at the quantum level. We present results for the level structure of black holes. We find a "principal series" of levels, corresponding to quantization of the area of the horizon. From each level of this principal series starts a quasi-continuum of levels due to excitations of the membrane. We discuss the statistical origin of the black hole entropy and the relation with Hawking radiation and with the information loss problem. The limits of validity of the membrane approach turn out to coincide with the known limits of validity of the thermodynamical description of black holes.
\end{abstract}




\section{Introduction}

In the search of a quantum theory of gravitation black holes might play a role similar to the hydrogen atom in quantum mechanics: they might be the system in which important conceptual problems and possibly even contradictions arise in a sharp form, and from which we can start to understand what are the "rules of the game" when both quantum mechanics and general relativity play an important role. An important problem arising in this context is the information loss paradox raised by Hawking [1], which apparently suggests that the evolution of states in a black hole background violates such a basic principle as unitarity. A closely related issue is the origin of the black hole entropy: the relation between the entropy of a black hole and the area of the horizon, $S=A / 4$ in Planck units, suggests the existence of a large number of microstates (on the order of $\sim \exp \left\{4 \pi M^{2}\right\}$ for a Schwarzschild black hole of mass $M$ ), of which there is no trace in the classical description. Another, less discussed, but possibly very important issue is the relation between black holes and elementary particles; 't Hooft [2] has proposed that the two concepts should merge as we cross the Planck mass (see also [3]).

The hearth of the problem is that we do not know what is a black hole at the quantum level. Indeed, it is not even clear what are the degrees of freedom to be quantized. Various authors, from early work of Bekenstein [4] to more recent works of 't Hooft [2] and Susskind, Thorlacius and Uglum [5] have suggested that the black hole is a normal quantum system with discrete energy levels. But it is far from clear what is the "Schroedinger equation" for a black hole, if any, or whether it can be described by a wave function.

A first important hint for understanding what are the degrees of freedom to be quantized comes from the membrane paridigm [6], in which the interaction of a classical black hole with the external environment, as seen by fiducial observerst, is entirely described in terms of a fictitious membrane located close to the horizon and endowed with physical properties like electric conductivity, temperature and viscosity.

At the quantum level, the description of the black hole horizon as a quantum surface with approximately one degree of freedom per Planck unit of area has been advocated by 't Hooft [2, 7], and it is strongly suggested by

\footnotetext{
${ }^{1}$ We will hereafter refer to observers external to the black hole and static with respect to it as "fiducial" observers. The precise definition of fiducial observers for both non-rotating and rotating black holes is given in ref. [6].
} 
the proportionality between the entropy and the area of the horizon.

A possible objection to such a description is that, although from the point of view of a fiducial observer the black hole behaves as if there were a membrane on the horizon, such a membrane certainly does not exist for the free falling observer. Then, it is not clear what is the meaning of assigning actual microscopic degrees of freedom to the membrane. Furthermore, in order to have a useful description it turned out [6] to be necessary to move the membrane a bit outside the horizon, defining the so-called stretched horizon. This allows to get rid of the details of the infalling matter and regularizes the infinite red-shift factor between the horizon and infinity. The amount of stretching is however quite arbitrary, so that the position of the membrane is determined by our "regularization" procedure, rather than by physics.

The answer to the first objection follows from the recent works [5, 8, 9, 10], where a principle of "black hole complementarity" is proposed. The principle states that the points of view of the free falling and fiducial observers are complementary, in a sense close to Bohr complementarity: even if an ideal "superobserver", who can compare the measurements of both the fiducial and the free falling observers, would conclude that their observations are actually contradictory, still no real contradiction arises because such superobservers cannot be realized in Nature. More precisely, the analysis of several Gedanken experiments [9] indicates that any attempt to compare the results of the measurements of a free falling observer crossing the horizon and of a fiducial observer turns out to involve assumptions on physics beyond the Planck scale. Similar considerations have been made in ref. [11, where it is argued that the commutators of the operators measuring Hawking radiation with operators inside the horizon grow uncontrollably large and prevent simultaneous measurements of the relative observables. This suggestion has been elaborated in the very recent paper [12].

This removes the first objection to the membrane approach. The membrane actually exists for the fiducial observer, who can legitimately assign it microphysical degrees of freedom and try to study the dynamics of this microscopic structure. The membrane is not detected by the free falling observer who is crossing the horizon, but this does not lead to any logical contradiction.

The second objection, concerning the arbitrariness in the position of the stretched horizon, can be answered taking into account the generalized uncer- 
tainty principle in quantum gravity, which assigns to the horizon a physical thickness because of quantum fluctuations [13].

The principle of black hole complementarity has deep physical consequences which imply a radical change in our view of space-time. In particular, as discussed by Susskind [10], it implies that even the notion of invariant event cannot be anymore relied upon. It seems to us that, if the ideas suggested by 't Hooft and by Susskind and collaborators will prove to be correct, they will represent a major conceptual advance in our understanding of quantum gravity.

If we accept the point of view of black hole complementarity, the membrane is the natural candidate for the location of the microstates of the black hole and the degrees of freedom of the membrane are interpreted as the variables to be quantized.

In this paper, in order to test these ideas, we carry them a bit further and propose a specific dynamical framework for the membrane. More precisely, we wish to explore the consequences of the following assumption: from the point of view of a fiducial observer, a black hole is described by a membrane whose dynamics is governed by an action principle. The action is taken to be that of the closed relativistic bosonic membrane in $D=4$ dimensions, which is proportional to the world-volume swept.

In sect. 2 we will show that this description makes sense at the classical level. While it is known that no stable classical solution of this action exists in flat space, we will find that in the external, fixed, background of a black hole (or, more in general, in metrics with a horizon, as in Rindler space) there is a stable classical solution which lies just on the horizon and other classical solutions, whose motion is confined to the region outside the horizon and which approach the horizon in a logarithmically divergent time. This result, independently of the applications to black hole physics, might be of interest in itself in the study of relativistic membranes.

We will then proceed to a quantum description (sect. 3). At this stage, we will have to resort to an approximation in which the only degree of freedom retained is the radius of the membrane, which is taken to have spherical symmetry. Some aspects of black hole physics, like the value of the entropy, are irretrievably lost in this approximation, since we are drastically reducing the number of degrees of freedom; however, many interesting features survive, and therefore it is a useful starting point, at least for qualitative understanding. The great advantage of the approximation is that the problem is reduced 
to an ordinary problem of quantum mechanics, rather than of quantum field theory. We will find that an interesting and plausible quantum structure emerges, both for Schwarzschild and more general Reissner-Nordstrom black holes. In the Rindler case, we will find that the dynamics of the membrane is governed by Liouville quantum mechanics. We will discuss the limits of validity of the membrane approach (sect. 3.3) and, remarkably, we will find that they coincide with the limits of validity of the thermodynamical description of black holes found in ref. [14].

Using these results, in sect. 4 we will be able to propose an energy level structure for black holes.

We will be mainly concerned with static properties, in particular with the energy level structure; however, we will also make some comments on the transition amplitudes between these levels, and their relation with Hawking radiation (sect. 5).

It should be stressed at this point that, while the mathematical formalism that we use is well-defined, the physical interpretation of our results, at the present stage, is only tentative. This is unavoidable in a situation in which we are trying to unravel what the "rules of the game" are, and what physical concepts are appropriate for a quantum description of black holes. Thus, our results can only be considered a possible starting point for further investigations.

At the appropriate points we will discuss the limits of validity of our computations. However, we want to stress now that the membrane approach is not, at our present level of understanding, a fundamental description of black holes. We rather think to it as an effective description, valid in a domain to be determined below. In some sense, the situation is similar to the large distance effects in $Q C D$, where the quark-antiquark potential at scales of order of $1 \mathrm{fm}$ is described, at the effective level, in terms of a string. Of course, this string is a useful description only on a given scale of distances: if we look more closely, there is no string, but just quarks and gluons. This means that we should not worry of the fact that the quantum theory of a purely bosonic membrane can be defined consistently only (and at most) in $D=27$ dimensions [15] (as bosonic strings require $D=26$ ), nor of the fact that, unlike string theory, a membrane theory is not renormalizable by power counting. For the same reason, the membrane tension which will be introduced is not a fundamental constant, but, as the string tension in $Q C D$, it is in principle derivable from the underlying theory. 
In this paper we will only study black holes with zero angular momentum. Although we believe that there is no fundamental difficulty in generalizing our approach to Kerr black holes, in this paper we will make use of the technical simplifications due to spherical symmetry.

\section{Classical membrane dynamics}

The action of the relativistic bosonic membrane is given by

$$
S=-\mathcal{T} \int d^{3} \xi \sqrt{-\operatorname{det}\left(g_{\mu \nu} \partial_{i} x^{\mu} \partial_{j} x^{\nu}\right)}
$$

where $\mathcal{T}$ is the membrane tension, and has dimensions of (mass $)^{3}$. The membrane world-volume is parametrized by $\xi^{i}=\left(\tau, \sigma_{1}, \sigma_{2}\right)$, that is $x^{\mu}=x^{\mu}(\xi)$. The indices $i, j$ take values $0,1,2$ and $\partial_{i}=\partial / \partial \xi^{i}$. The metric of the target space is $g_{\mu \nu}$, with $\mu, \nu=0,1,2,3$ and the signature is Minkowskian. We use Planck units, $\hbar=c=G=1$. The action (看) is the natural generalization of the relativistic action of a spinless pointlike particle, which is proportional to its world-line, and of a bosonic string, which is proportional to the world-area swept.

This action or modifications of it have been considered in various physical problems. It was first proposed by Dirac [16] in 1962 as a possible model for an extended electron. Later it has been studied both as a generalization of bosonic strings and with motivations coming from bag models of hadrons 117 , 18]. In recent years it has been used to investigate the bubble nucleation process in the early Universe in inflationary cosmology [19, 20], while its supersymmetric extension ("supermembrane") received some attention as a generalization of superstrings [21, 22].

For pointlike particles and strings, the square root can be eliminated introducing an einbein or a world-sheet metric, respectively; similarly, here one can introduce a world-volume metric $h_{i j}, i, j=0,1,2$. At the classical level, the action given in eq. (11) is equivalent [23] to the action

$$
S=-\frac{\mathcal{T}}{2} \int d^{3} \xi \sqrt{-h}\left[h^{i j} g_{\mu \nu} \partial_{i} x^{\mu} \partial_{j} x^{\nu}-1\right],
$$

where $h=\operatorname{det} h_{i j}$. Two differences with the string case are noteworthy. First, the appearance of a cosmological term in eq. (2). Second, the fact that the metric $h_{i j}$ cannot be gauged away even at the classical level. 
The action (2) is invariant under general coordinate transformations of the world-volume. The equations of motion obtained with variation with respect to $h_{i j}$ are

$$
h_{i j}=g_{\mu \nu} \partial_{i} x^{\mu} \partial_{j} x^{\nu} .
$$

Thus $h_{i j}$ is the metric induced by $g_{\mu \nu}$ on the surface of the membrane. Variation with respect to $x^{\mu}$ gives

$$
\partial_{i}\left(\sqrt{-h} h^{i j} g_{\mu \nu} \partial_{j} x^{\nu}\right)-\frac{1}{2} \sqrt{-h} h^{i j} g_{\rho \sigma, \mu} \partial_{i} x^{\rho} \partial_{j} x^{\sigma}=0 .
$$

In order to find solutions of the equations of motion with spherical symmetry one proceeds as follows [17]. One introduces "polar fields", i.e. a set of three fields $r(\xi), \theta(\xi), \phi(\xi)$ (where $\xi$ is a shorthand for $\left(\tau, \sigma_{1}, \sigma_{2}\right)$ ) defined by

$$
x^{\mu}=\left(x^{0}, r \sin \theta \cos \phi, r \sin \theta \sin \phi, r \cos \theta\right) .
$$

Next one fixes the gauge

$$
x^{0}(\xi)=\tau, \quad \theta(\xi)=\sigma_{1}, \quad \phi(\xi)=\sigma_{2} .
$$

This gauge fixing is useful also at the quantum level, since in this gauge there is no Faddeev-Popov ghost 24]. The parameters $\sigma_{1}, \sigma_{2}$ satisfy

$$
0 \leq \sigma_{1} \leq \pi, \quad 0 \leq \sigma_{2} \leq 2 \pi .
$$

One now makes the spherical ansatz, $r\left(\tau, \sigma_{1}, \sigma_{2}\right)=r(\tau)$. This reduces the problem to a single degree of freedom $r(\tau)$. Of course, at the classical level this simply means that we are looking for special solutions of the equations of motion, but the solutions we get are exact. At the quantum level, instead, restriction to membranes of this form is a drastic approximation, analogous to the minisuperspace approximation used in quantum cosmology. However, in the case of black holes, the no-hair theorem ensures that at the classical level the horizon has spherical symmetry, so we expect that the approximation might be useful even in the quantum theory.

In any application, an especially important issue is the existence of stable classical solutions. The classical equations of motion of the action (2) have been studied in flat target space, $g_{\mu \nu}=\eta_{\mu \nu}$, in refs. [17, 25]. In this case, no stable classical solution has been found. The membrane contracts under its self-attraction, until it collapses. While strings can stabilize themselves 
rotating, this is not possible for a membrane in $D=4$, since the centrifugal force will be zero on the rotation axis (stable rotating classical solutions exist in dimensions higher than four [26]). Alternatively, one can study the equations on spaces with non trivial topology, such as $S^{2} \times R^{D-2}$, where stable solutions exist [22, 24].

In the Dirac extended electron model, instead, the self attraction is balanced by the electrostatic repulsion, giving rise to a stable solution. However, the model is unstable under quadrupole deformations from spherical symmetry 18 .

The equations of motion have also been studied in the case of a selfgravitating membrane [19, 20, 27], in which the metric is generated by the membrane itself, so that it is taken to be a Reissner-Nordstrom metric outside the membrane and a flat metric inside, with suitable junction conditions. We will instead be interested in the motion of a membrane in a background metric which is fixed from the beginning (it is generated by the matter which has collapsed to form the black hole) and in this section we only consider the situation in which the back-reaction of the membrane on the metric is neglegible. The limits of this approximation will be discussed in sect. 3.1.

We will find that stable solutions of the action given in eq. (11) exist when the metric $g_{\mu \nu}$ is taken to be a metric with a horizon. Let us now analyze separately the Reissner-Nordstrom and Rindler cases.

\subsection{Reissner-Nordstrom metric}

We consider a general Reissner-Nordstrom black hole with mass $M$ and charge $Q$. We use Boyer-Lindquist coordinates, which are appropriate for a fiducial observer, and we only consider the metric outside the horizon. As we will see, this approach is consistent since, for the fiducial observer, a membrane initially outside the horizon always remains outside.

The Reissner-Nordstrom metric is given by

$$
\begin{gathered}
d s^{2}=-\alpha d t^{2}+\alpha^{-1} d r^{2}+r^{2}\left(d \theta^{2}+\sin ^{2} \theta d \phi^{2}\right), \\
\alpha=1-\frac{2 M}{r}+\frac{Q^{2}}{r^{2}}=\frac{1}{r^{2}}\left(r-r_{+}\right)\left(r-r_{-}\right),
\end{gathered}
$$

and $r_{ \pm}=M \pm \sqrt{M^{2}-Q^{2}}$. The outer horizon is at $r=r_{+}$. The induced 
metric is computed from eq. (3) and in the gauge $x_{0}\left(\tau, \sigma_{1}, \sigma_{2}\right)=\tau$ is

$$
h_{i j}=\left(\begin{array}{ccc}
-\left(\alpha^{2}-\dot{r}^{2}\right) / \alpha & & \\
& r^{2} & \\
& & r^{2} \sin ^{2} \theta
\end{array}\right)
$$

where $\dot{r}=d r / d \tau$. Using the ansatz (5), the equation of motion (4) with $\mu=0$ gives immediately

$$
\partial_{0}\left[r^{2} \alpha\left(\alpha-\frac{\dot{r}^{2}}{\alpha}\right)^{-1 / 2}\right]=0 .
$$

The equations with $\mu=1,2,3$ give a single second order differential equation

$$
r \ddot{r}+2\left(\alpha^{2}-\dot{r}^{2}\right)+\frac{r \alpha^{\prime}}{2 \alpha}\left(\alpha^{2}-3 \dot{r}^{2}\right)=0,
$$

where $\alpha^{\prime}=d \alpha / d r$. Eq. (11) is the first integral of eq. (12), except that eq. (11) also admits the solution $\dot{r}=\ddot{r}=0, r=$ arbitrary constant, while in eq. (12) this is a solution only if this arbitrary constant is equal to the horizon radius (so that $\alpha=0$ ). Then, the equation governing the radial motion is eq. (12), or eq. (11) with the solution $r=r_{0}$ removed unless the constant $r_{0}$ is equal to $r_{+}$. Therefore, we have found that there is a stable static solution corresponding to a spherical membrane lying on the horizon.

Integration of eq. (11) gives

$$
\dot{r}^{2}=\alpha^{2}-C^{2} r^{4} \alpha^{3}
$$

where $C$ is an integration constant fixed by the initial conditions. (Note that timelike motions outside the horizon satisfy the condition $\alpha^{2}-\dot{r}^{2}>$ $0)$. Eq. (13) is formally identical to the motion of a non-relativistic point particle with unit mass and zero energy in the potential $U(r)=\left(-\alpha^{2}+\right.$ $\left.C^{2} r^{4} \alpha^{3}\right) / 2, r>r_{+}$(see fig. 1). Depending on the sign of $\dot{r}(0)$, the membrane can expand until it reaches a maximum value and then recontract, or it will contract immediately. In both cases, it reaches asymptotically $r=r_{+}$, in a logarithmically divergent time (or in a linearly divergent time for extremal black holes, $M=Q$ ). This asymptotic approach to the horizon is just what we expect for any object, as seen by a fiducial observer.

Note that we never need to know the metric inside the "nominal" horizon, $r=r_{+}$. A membrane initially at $r>r_{+}$will always remain outside, and a 


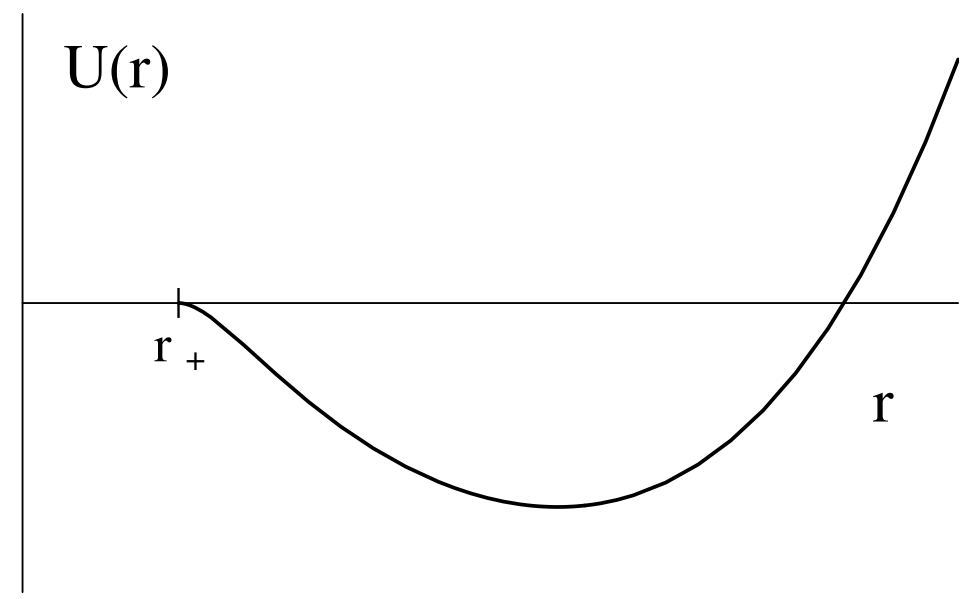

Figure 1: The effective potential discussed in the text. The classical motion of the spherical membrane is formally equivalent to the motion of a particle with $E=0$ in this potential.

fiducial observer can consistently describe the dynamics of the membrane without making any reference to the region $r<r_{+}$. Of course, this is not surprising; it is just what happens for any object, pointlike or extended, from the point of view of a fiducial observer.

If we substitute the spherical ansatz (5) into the membrane action, eq. (2), with the induced metric given by eq. (3) and integrate over the variables $\sigma_{1}, \sigma_{2}$, we find the action

$$
S=-4 \pi \mathcal{T} \int d \tau r^{2}\left(\alpha-\frac{\dot{r}^{2}}{\alpha}\right)^{1 / 2}
$$

The Euler-Lagrange equation of this action is eq. (12). Thus, eq. (14) is the effective action for the radial coordinate.

It is interesting to study the stability of the solution that we have found under non-spherical deformations. Using again the gauge fixing (6) but keeping $r\left(\tau, \sigma_{1}, \sigma_{2}\right)$ generic, from eq. (4) with $\mu=0$ we immediately find the more general equation

$$
\dot{r}^{2}(\tau, \theta, \phi)=\alpha^{2}-C^{2}(\theta, \phi) r^{4} \alpha^{3},
$$


and $C$ is the integration constant, whose dependence on $\theta, \phi$ is determined by the dependence of $r$ and $\dot{r}$ on $\theta, \phi$ at the initial time $\tau=\tau_{0}, r\left(\tau_{0}, \theta, \phi\right)=$ $r_{0}(\theta, \phi)$. We see that, as the horizon is approached, $\alpha \rightarrow 0$ and the $C$ dependent term in eq. (15) become less and less important. If at $\tau=0$ the membrane is sufficiently close to the horizon, the subsequent evolution is given, for a Schwarzschild black hole, by

$$
r(\tau, \theta, \phi) \simeq 2 M+\left(r_{0}(\theta, \phi)-2 M\right) \exp \{-\tau /(2 M)\},
$$

and any initial deviation from spherical symmetry is washed out in an exponentially small time. This is exactly the behavior expected for a black hole from the classical no-hair theorem.

In the above discussion, the equations of motion have been studied in terms of a field $r\left(\tau, \sigma_{1}, \sigma_{2}\right)$ which, by definition, is constrained to satisfy $r \geq 0$. Furthermore, we are only considering the equations of motion in the region $r \geq r_{+}$. When we have in mind to proceed to a quantum field theory, such a field is not appropriate, since a constraint of the form $r \geq 0$ (or $r \geq r_{+}$) is very difficult to implement and has quite non trivial effects on the dynamics. We therefore introduce a tortoise field $r_{*}(\xi)$, analogous to the standard tortoise coordinate and defined by

$$
d r_{*}=\frac{d r}{\alpha} .
$$

In particular for a Schwarzschild black hole, with an appropriate choice for the integration constant in eq. (17),

$$
r_{*}=r+2 M \log \frac{r-2 M}{2 M} .
$$

The tortoise field defined by eq. (17) ranges from $-\infty$ to $+\infty$ as $r$ goes from the horizon, $r=r_{+}$, to infinity. We can rewrite the equations of motion in terms of the field $r_{*}\left(\tau, \sigma_{1}, \sigma_{2}\right)$, making the ansatz that $r_{*}$ actually depends only on $\tau$. Eq. (13) then becomes

$$
\dot{r}_{*}^{2}=1-C^{2} r^{4} \alpha
$$

where now $r$ is a function of $r_{*}$ given implicitly by eq. (17) (or by eq. (18) for a Schwarzschild black hole). In terms of $r_{*}$ there is no static solution, since the solution $r=r_{+}$has been mapped to $r_{*}=-\infty$. Rather, at large values of $\tau$ the solution is approaching $r_{*}=-\infty$ asymptotically. 


\section{$2.2 \quad$ Rindler metric}

We now consider the Rindler metric, which is the metric appropriate to an observer with constant acceleration $g$ in flat Minkowski space. Let $(T, x, y, Z)$ be the Minkowski coordinates. If we define new coordinates $t, z$ from

$$
\begin{aligned}
& T=z \sinh g t \\
& Z=z \cosh g t,
\end{aligned}
$$

the flat Minkowski metric takes the form

$$
d s^{2}=-g^{2} z^{2} d t^{2}+d x^{2}+d y^{2}+d z^{2} .
$$

This spacetime has an horizon at $z=0$. Besides its intrinsic interest, this metric is the limit of the Schwarzschild metric for large black hole masses, after an appropriate change of variables. In fact, defining

$$
x=2 M\left(\theta-\frac{\pi}{2}\right), \quad y=2 M \phi, \quad z=4 M \sqrt{1-\frac{2 M}{r}},
$$

and identifying $g=1 /(4 M)$, the Schwarzschild and Rindler metrics differ only by terms $O\left((z / M)^{2}\right), O\left((x / M)^{2}\right)$. The region $z \geq 0$ is mapped in the region $r \geq 2 M$. The fact that the spatial metric is flat has a number of simplifying features which makes this metric particularly useful for our analysis. We will consider membranes in the region $z \geq 0$, and at the classical level we look for a solution of the equations of motion for the four fields $x^{\mu}\left(\tau, \sigma_{1}, \sigma_{2}\right)$ of the form

$$
\begin{array}{ll}
t\left(\tau, \sigma_{1}, \sigma_{2}\right)=\tau & x\left(\tau, \sigma_{1}, \sigma_{2}\right)=\sigma_{1} \\
z\left(\tau, \sigma_{1}, \sigma_{2}\right)=z(\tau) & y\left(\tau, \sigma_{1}, \sigma_{2}\right)=\sigma_{2} .
\end{array}
$$

Inserting the ansatz (23) in the equations of motion we find the induced metric

$$
h_{i j}=\left(\begin{array}{ccc}
-g^{2} z^{2}+\dot{z}^{2} & & \\
& 1 & \\
& & 1
\end{array}\right) .
$$

The equations of motion (4) with $\mu=1,2$ are identically satisfied, while the equations with $\mu=0,3$ give a single equation for $z(\tau)$,

$$
z \ddot{z}-2 \dot{z}^{2}+g^{2} z^{2}=0
$$

\footnotetext{
${ }^{2}$ As in the black hole case, the $\mu=0$ equation has also a solution $\dot{z}=0$ which is not shared by the $\mu=3$ equation, unless $z=0$.
} 
As in the black hole case, we find a stable static solution on the horizon, $\dot{z}=\ddot{z}=z=0$. The motion with arbitrary initial conditions can be easily studied from eq. (25), which can be integrated analytically. If the initial condition is $z(0)=z_{0}, \dot{z}(0)=\dot{z}_{0}$, and we define $v_{0}=\dot{z}_{0} /\left(g z_{0}\right)$ (which is the initial velocity measured in proper time units, and satisfies $\left.\left|v_{0}\right| \leq 1\right)$, the solution is

$$
z(\tau)=\frac{z_{0}}{\cosh g \tau-v_{0} \sinh g \tau} .
$$

The qualitative features of the motion are analogous to the black hole case. Substituting the ansatz (23) in the membrane action and defining $\mathcal{T}^{\prime}=$ $\mathcal{T}\left(\int d x d y\right)$, we find the effective action

$$
S=-\mathcal{T}^{\prime} \int d \tau\left(g^{2} z^{2}-\dot{z}^{2}\right)^{1 / 2}
$$

The Euler-Lagrange equation of this action is just eq. (25).

\section{Quantum membrane dynamics}

At the classical level, our approach has given satisfactory results. We have found classical solutions in correspondence with horizons, and the classical dynamics is certainly compatible with the black hole interpretation that we are trying to develop. Of course, the motivation of our investigation is to study black holes at the quantum level. We then proceed to the quantum description.

The quantization of the full membrane action (2) is a very difficult task. We will therefore make a drastic approximation: we will limit ourselves to spherical membranes, $r=r(\tau)$, in the black hole case, and to membranes of the form $z=z(\tau)$ in Rindler space. This approximation is analogous to the minisuperspace approximation used in quantum cosmology. It has already been used in refs. [19, 20] in the study of the quantum dynamics of selfgravitating membranes and of membranes in flat space. Having reduced the number of degrees of freedom, we are giving up the possibility of computing the black hole entropy. However, we will see that many interesting feature are still present in this approximation.

Our starting point, therefore, is the action (14) in the black hole case and the action (27) in Rindler space. Of course, when we proceed to a quantum 
treatement, we must also ask under what conditions the loop corrections to the action (1) or to the action (2) (which in general are not equivalent at the quantum level) are under control. We will discuss this point in sect. 3.3.

\subsection{Reissner-Nordstrom metric}

In this case the Lagrangian is

$$
L=-\mathcal{T}^{\prime} r^{2}\left(\alpha-\frac{\dot{r}^{2}}{\alpha}\right)^{1 / 2}
$$

where we have defined $\mathcal{T}^{\prime}=4 \pi \mathcal{T}$. To quantize the system, we pass to a Hamiltonian description. One can try to proceed defining the conjugate momentum $p$

$$
p=\frac{\delta L}{\delta \dot{r}}=\mathcal{T}^{\prime} \frac{r^{2} \dot{r}}{\alpha}\left(\alpha-\frac{\dot{r}^{2}}{\alpha}\right)^{-1 / 2} .
$$

This equation can be inverted to give $\dot{r}$ as a function of $p$, and the Hamiltonian $H=p \dot{r}-L$ can be computed. We find

$$
H=\sqrt{\alpha^{2} p^{2}+\mathcal{T}^{\prime 2} r^{4} \alpha} .
$$

The square root is not a problem, since the expression inside the square root is positive definite outside the horizon. However, when we go over to the quantum theory and impose canonical commutation relations between $r$ and $p$, eq. (30) is beset by a very severe ordering ambiguity because of the term $\alpha^{2} p^{2}$, and thus it is not suitable as a starting point for quantization. Furthermore, when we have in mind that our quantum mechanical problem is a first approximation to a field theoretical problem, then $r$ is not the most appropriate variable since, as we mentioned above, the field $r(\xi)$ is subject to the condition $r \geq 0$ by definition, and to the condition $r>r_{+}$if we want to set up a quantum description of the black hole which does not make reference to the black hole interior, as it is implicit in the membrane approach, and such conditions are difficult to implement in a field theory. Thus neither the variable $r$ nor the Hamiltonian (30) are appropriate.

Remarkably, we have found that the introduction of the tortoise field, eq. (17), which solves the problem of the constraint $r>r_{+}$, also gives a very natural solution of the ordering ambiguity. We define the momentum 
conjugate to $r_{*}$,

$$
p_{*}=\frac{\delta L}{\delta \dot{r}_{*}}=\mathcal{T}^{\prime} \alpha^{1 / 2} r^{2} \dot{r}_{*}\left(1-\dot{r}_{*}^{2}\right)^{-1 / 2} .
$$

For the Hamiltonian, $H=p_{*} \dot{r}_{*}-L$, we get

$$
H=\sqrt{p_{*}^{2}+\mathcal{T}^{\prime 2} r^{4} \alpha},
$$

which does not present any ordering ambiguity. In eq. (32) $r$ is a function of $r_{*}$ given implicitly by eq. (17). We now quantize the system imposing

$$
\left[r_{*}, p_{*}\right]=i
$$

and describe the quantum membrane with a wave function $\psi\left(r_{*}\right)$ which gives the amplitude of finding the membrane at the given value $r_{*}$. The Schroedinger equation for the black hole is then $H \psi_{E}=E \psi_{E}$ with $H$ given in eq. (32). At the level of first quantization this is equivalent to

$$
\left(-\frac{d^{2}}{d r_{*}^{2}}+\mathcal{T}^{\prime 2} r^{4} \alpha\right) \psi_{E}\left(r_{*}\right)=E^{2} \psi_{E}\left(r_{*}\right) .
$$

This equation can be interpreted as a Schroedinger equation in one dimension for a particle with mass $m=1 / 2$ in a potential

$$
V\left(r_{*}\right)=\mathcal{T}^{\prime 2} r^{4} \alpha(r),
$$

with $r=r\left(r_{*}\right)$ given by eq. (17), and with $E^{2}$ playing the role of the eigenvalue. But of course, one recognizes immediately that the origin of eq. (34) must be an equation of the Klein-Gordon type. In fact, Aurilia and Spallucci [20] have derived the analogous Klein-Gordon equation in the case of flat space. In our derivation of the Lagrangian (28) we have chosen the gauge $x^{0}\left(\tau, \sigma_{1}, \sigma_{2}\right)=\tau$. If we do not fix the gauge, but work with $x^{0}$ generical, we find instead the Lagrangian

$$
L=-\mathcal{T}^{\prime} r^{2}\left[\alpha\left(\frac{d x^{0}}{d \tau}\right)^{2}-\frac{\dot{r}^{2}}{\alpha}\right]^{1 / 2} .
$$

\footnotetext{
${ }^{3}$ Actually, an ambiguity still exists connected with the arbitrary constant in the integration of eq. (17). We will discuss it carefully in sect. 3.3, where we will find that it fixes the limit of validity of the membrane description.
} 
Defining the momenta conjugate to $r_{*}$ and $x^{0}$,

$$
\begin{aligned}
& p_{0}=\frac{\delta L}{\delta \dot{x}^{0}}=-\mathcal{T}^{\prime} r^{2} \alpha^{1 / 2} \dot{x}^{0}\left[\left(\dot{x}^{0}\right)^{2}-r_{*}^{2}\right]^{-1 / 2} \\
& p_{*}=\frac{\delta L}{\delta \dot{r}_{*}}=+\mathcal{T}^{\prime} r^{2} \alpha^{1 / 2} \dot{r}_{*}\left[\left(\dot{x}^{0}\right)^{2}-r_{*}^{2}\right]^{-1 / 2}
\end{aligned}
$$

we see that they satisfy the relation $p_{0}^{2}-p_{*}^{2}=\mathcal{T}^{\prime 2} r^{4} \alpha$, from which it follows the equation

$$
\left(-\frac{\partial^{2}}{\partial t^{2}}+\frac{\partial^{2}}{\partial r_{*}^{2}}\right) \phi\left(r_{*}, t\right)=\mathcal{T}^{\prime 2} r^{4} \alpha \phi\left(r_{*}, t\right) .
$$

In the flat space limit, $\alpha \rightarrow 1, r_{*} \rightarrow r$, we recover the corresponding equation found in ref. [20].

As long as we are interested in the first quantization of the membrane the two approaches, based respectively on the Schroedinger equation, eq. (34), or on the Klein-Gordon equation, eq. (39), are equivalent. In the following we shall use the approach based on the Schroedinger equation, which is intuitively appealing. If one studies the second quantization of the membrane in the minisuperspace approximation (which is beyond the scope of this paper) eq. (39) is the correct starting point.

The potential $V\left(r_{*}\right)$ is plotted in fig. 2. We see that $V\left(r_{*}\right)$ also illustrates nicely the classical motion. The stable classical solution is at $r_{*}=-\infty$, and a membrane with given energy, depending on whether $\dot{r}(0)$ is positive or negative, can expand up to a maximum radius and recontract, or it can immediately contract and approach the horizon asymptotically.

Let us now examine the qualitative features of the Schroedinger equation in the potential $V\left(r_{*}\right)$. The fact that $V\left(r_{*}\right) \rightarrow \infty$ as $r_{*} \rightarrow \infty$ is very welcome. It means that, independently of the value of the excitation energy $E$, the wave function of the black hole vanishes exponentially for sufficiently large values of $r_{*}$. In particular, no tunneling of the membrane to infinity is possible, which would have made our description quite unreasonable. (Or at least very dangerous, since it would have implied that a black hole has a small but finite probability of expanding until it covers the whole Universe!)

\footnotetext{
${ }^{4}$ Depending on the spin, the square root can also be eliminated in favor of a Dirac equation, see ref. 28] for a similar treatement in flat space. However, we are only considering black holes with $\mathbf{J}=0$.
} 


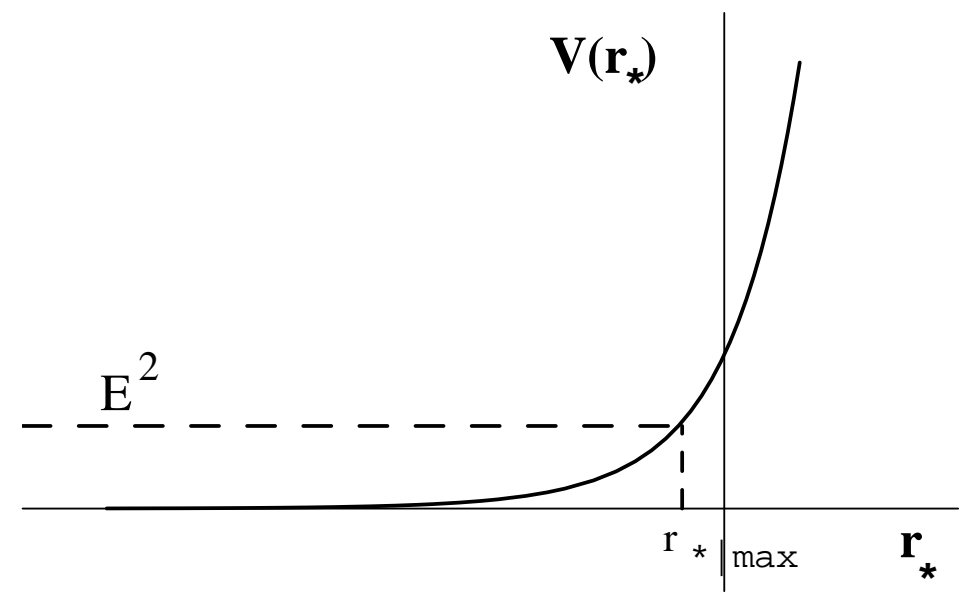

Figure 2: The effective potential $V\left(r_{*}\right)$ vs. $r_{*}$. The wave function with energy $E$ decreases exponentially for $r_{*}>r_{* \mid \max }$.

The fact that $V\left(r_{*}\right) \rightarrow 0$ as $r_{*} \rightarrow-\infty$, and therefore the existence of a continuous set of energy levels, is also welcome. It means that the black hole can absorbe arbitrarily small quanta of energy. This point, however, is susceptible of a small but important modification. If we introduce the stretched horizon [6, 用, we limit the range of $r_{*}$ to $r_{*}>-L$, where $L$ is a large but finite constant. The energy levels then become discrete, although very finely spaced. This point will be discussed in sect. 1 .

Since the metric $g_{\mu \nu}$ has been fixed at the beginning, this quasi-continuum of levels belongs to a black hole with a given, fixed, mass $M$ and charge $Q$, and hence with fixed area of the horizon. Thus, we consider them as excitations of a black hole with a given area. Actually, an excited level with excitation energy $E$ has a larger value of the classical horizon radius, determined graphically in fig. 2 from the condition $E^{2}=V\left(r_{* \mid \max }\right)$. Of course, if $E$ is sufficiently large the corresponding value of $r_{* \mid \max }$, and hence of the horizon area, can be made arbitrarily large. However, in this limit our membrane approach is not self-consistent, since we have neglected any back reaction of the membrane on the metric. Consider instead values of the 
excitation energy restricted by the condition

$$
E^{2} \ll \mathcal{T}^{\prime 2} \kappa r_{+}^{3},
$$

where the surface gravity of a black hole, $\kappa$, is defined as

$$
\kappa=\left.\frac{1}{2} \frac{d \alpha}{d r}\right|_{r=r_{+}}=\frac{r_{+}-r_{-}}{2 r_{+}^{2}} .
$$

Let $r_{h}$ be the value of $r$ corresponding to $r_{* \mid \max }$. From the explicit expression of the potential, eq. (35), we get

$$
r_{h} \simeq r_{+}+\frac{E^{2}}{2 \mathcal{T}^{\prime 2} \kappa r_{+}^{4}}
$$

so that $r_{h}-r_{+} \ll 1$ because of eq. (40) and because $r_{+} \gg 1$. For the variation of the area of the horizon we get

$$
\Delta A=4 \pi\left(r_{h}^{2}-r_{+}^{2}\right) \simeq \frac{4 \pi E^{2}}{\mathcal{T}^{\prime 2} \kappa r_{+}^{3}} \ll 1 .
$$

Thus, in this limit the variation of the area is much smaller than one Planck unit and, as we will argue in sect. 4, such a small variation is not observable. In this domain we expect that the approximation of neglecting the backreaction of the membrane is self-consistent. Furthermore, it is natural to consider all membrane levels restricted by eq. (40) as excitations of a black hole with a given, fixed, area of the horizon. This point will be discussed further in sect. 1 .

The above results means that, given the mass and the charge, the quantum state of a Reissner-Nordstrom black hole is not uniquely determined, contrarily to what happens in the classical description. There is still a large number of microstates, corresponding to the energy levels that we have found. This quasi-continuum of levels is not visible in the classical description of black holes, and the corresponding coarse-graining gives rise to the black hole entropy. We do not attempt to compute the entropy from our model, since, having frozen all degrees of freedom apart from the radial coordinate, the levels that we are discussing are only a (very small) subset of all black hole levels. However, it is quite clear that in a full treatement of the membrane dynamics the number of microstates must grow exponentially with the area. 
It is interesting to examine the form of the potential $V\left(r_{*}\right)$ for large negative values of $r_{*}$. From the definition, eq. (17), one finds that for large negative values of $r_{*}$ the relation between $r$ and $r_{*}$ is, for $\kappa \neq 0$,

$$
r-r_{+} \simeq \text { const. } e^{-2 \kappa\left|r_{*}\right|} .
$$

Then one finds immediately that

$$
\left.V\left(r_{*}\right) \simeq \mathcal{T}^{\prime} r_{+}^{4}\left(r-r_{+}\right) \frac{d \alpha}{d r}\right|_{r=r_{+}} \simeq \text { const. } e^{-2 \kappa\left|r_{*}\right|} \quad\left(r_{*} \rightarrow-\infty\right) .
$$

Even if in eq. (34) $V\left(r_{*}\right)$ formally plays the role of a potential, dimensionally it is rather a potential squared; thus, the "physical potential" $V_{\mathrm{ph}}=\sqrt{V}$ close to the horizon is a Yukawa potential corresponding to the exchange of a particle of mass $\kappa$. For extremal black holes, $Q=M, \kappa=0$. The relation between $r$ and $r_{*}$ is

$$
r_{*}=r+2 r_{+} \log \left(\frac{r-r_{+}}{\text {const. }}\right)-\frac{r_{+}^{2}}{r-r_{+}},
$$

so that as $r \rightarrow r_{+}, r_{*} \simeq-r_{+}^{2} /\left(r-r_{+}\right)$. The potential is

$$
\left.V\left(r_{*}\right) \simeq \mathcal{T}^{\prime 2} r_{+}^{4} \frac{1}{2}\left(r-r_{+}\right)^{2} \frac{d^{2} \alpha}{d r^{2}}\right|_{r=r_{+}} .
$$

Then, for $V_{\mathrm{ph}}$ we find a Coulomb potential,

$$
V_{\mathrm{ph}}\left(r_{*}\right) \simeq \frac{\mathcal{T}^{\prime} \mathrm{r}_{+}^{3}}{\left|r_{*}\right|} \quad\left(r_{*} \rightarrow-\infty\right) .
$$

The appearence of the parameter $\kappa$ in eq. (45) is quite suggestive of a relation with Hawking radiation, since $\kappa$ is related to Hawking temperature by $T_{H}=$ $\kappa /(2 \pi)$. We will discuss this point further in sect. 1 .

\subsection{Rindler metric}

In Rindler space we start with the Lagrangian eq. (27),

$$
L=-\mathcal{T}^{\prime}\left(g^{2} z^{2}-\dot{z}^{2}\right)^{1 / 2} .
$$


As in the black hole case, if we define a momentum $p$ conjugate to $z$ we find an Hamiltonian, $H=p z-L$, which is beset by ordering ambiguities:

$$
H=g z \sqrt{p^{2}+\mathcal{T}^{\prime 2}} \text {. }
$$

By analogy with black holes, we define a coordinate $z_{*}$ from $d z=g z d z_{*}$, or

$$
z_{*}=\frac{1}{g} \log \frac{z}{z_{0}}
$$

where $z_{0}$ is an arbitrary integration constant. Contrarily to the black hole case, the relation between $z_{*}$ and $z$ can be inverted analytically, $z\left(z_{*}\right)=$ $z_{0} \exp \left(g z_{*}\right)$. Then we define

$$
p_{*}=\frac{\delta L}{\delta \dot{z}_{*}}
$$

and the Hamiltonian $H=p_{*} z_{*}-L$ is

$$
H=\sqrt{p_{*}^{2}+\mathcal{T}^{\prime 2} g^{2} z^{2}}=\sqrt{p_{*}^{2}+\mu^{2} \exp \left\{2 g z_{*}\right\}},
$$

where we have defined $\mu=\mathcal{T}^{\prime} g z_{0}$. Note that the arbitrary constant $z_{0}$ only appears in the combination $z_{0} \mathcal{T}^{\prime}$, and therefore it is reabsorbed in a single constant $\mu$ which characterizes the membrane. This Hamiltonian does not have ordering ambiguities, and $z_{*}$ ranges between $-\infty$ and $+\infty$. The qualitative features of the potential $V\left(z_{*}\right)=\mu^{2} \exp \left\{2 g z_{*}\right\}$ are similar to the black hole case, and the same considerations apply here. However, in this case the Schroedinger equation can be solved exactly. The equation is

$$
\left(-\frac{d^{2}}{d z_{*}^{2}}+\mu^{2} e^{2 g z_{*}}\right) \psi_{E}\left(z_{*}\right)=E^{2} \psi_{E}\left(z_{*}\right) .
$$

This is the wave equation of Liouville quantum mechanics [29]. Introducing $u=(\mu / g) \exp \left(g z_{*}\right)=\mathcal{T}^{\prime} z$, which ranges between zero and infinity, the equation becomes

$$
\psi_{E}^{\prime \prime}+\frac{1}{u} \psi_{E}^{\prime}-\left(1-\frac{E^{2}}{g^{2} u^{2}}\right) \psi_{E}=0,
$$

where the prime denotes differentiation with respect to $u$. Eq. (55) is a Bessel equation of imaginary order. Taking into account the boundary condition $\psi=0$ at $u=\infty$, the solution is

$$
\psi_{E}(u)=N(E) K_{i \frac{E}{g}}(u)
$$


where $K$ is the MacDonald function and $N(E)$ is a normalization constant to be determined below. If $u \gg E / g, \psi_{E}(u)$ decreases exponentially

$$
\psi_{E}(u) \simeq N(E) \sqrt{\frac{\pi}{2 u}} e^{-u},
$$

while close to the horizon, $u \simeq 0$, the wave function, expressed again as a function of $z_{*}$, is a superposition of incoming and reflected waves,

$$
\psi_{E}\left(z_{*}\right) \simeq N(E) \frac{i g}{2 E}\left[\left(\frac{\mu}{2 g}\right)^{i E / g} \Gamma\left(1-i \frac{E}{g}\right) e^{i E z_{*}}-\left(\frac{\mu}{2 g}\right)^{-i E / g} \Gamma\left(1+i \frac{E}{g}\right) e^{-i E z_{*}}\right] .
$$

( $\Gamma$ is the Euler gamma function). For later considerations, it is important to compute also the normalization factor. If we use standard continuum energy normalization the result is 29

$$
N(E)=\left(\frac{\sqrt{2} \sinh (\pi E / g)}{\pi g}\right)^{1 / 2} .
$$

Another possible normalization is suggested by the fact that in curved space (if $g_{00}$ is time-independent) a continuity equation takes the form (see e.g. ref. [6], eq. (3.46))

$$
\sqrt{-g_{00}} \frac{\partial j^{0}}{\partial \tau}=-\nabla \cdot\left(\sqrt{-g_{00}} \mathbf{j}\right)
$$

where $\tau$ is the proper time and $j^{\mu}=\left(j^{0}, \mathbf{j}\right)$ is a conserved current. The integrated form, for $j^{0}=|\psi|^{2}$, is

$$
\frac{\partial}{\partial \tau} \int_{V} d^{3} V \sqrt{-g_{00}}|\psi|^{2}=-\oint_{\partial V} d \mathbf{s} \cdot \mathbf{j} \sqrt{-g_{00}}
$$

This suggests that another useful normalization of the wave function, in $(3+1)$ dimensions, is given by

$$
\int d^{3} x \sqrt{-g_{00}}|\psi|^{2}=1
$$

(We extend the integration only to the region outside the horizon.) In our case, the problem is one-dimensional and $g_{00}=-g^{2} z^{2}$. Thus, we require

$$
\int_{0}^{\infty} d z g z|\psi|^{2}=1
$$


This gives the normalization factor

$$
\tilde{N}(E)=\mathcal{T}^{\prime}\left(\frac{2 \sinh (\pi E / g)}{\pi E}\right)^{1 / 2} .
$$

In fig. 3 we plot the quantity $\rho$ defined as

$$
\rho(u)=\frac{1}{\mathcal{T}^{\prime}} \sqrt{-g_{00}}\left|\psi_{E}(u)\right|^{2},
$$

where $\psi_{E}$ is normalized with $\tilde{N}(E)$, so that $\int_{0}^{\infty} d u \rho(u)=1$. At small values

of $u$ the wave function has an oscillatory behavior, see eq. (58). If $E \lesssim g$ the amplitudes of these oscillations are very small compared to the heighest peak, and they are not visible in the scale of fig. 3a. For large values of $E$ they become quite visible, see fig. 3b.

\subsection{Limits of validity of the membrane description}

As we mentioned in the Introduction, the membrane approach should be considered as an effective rather than a fundamental description of black holes. We can now discuss the domain in which we expect such an effective description to be adequate.

The introduction of the tortoise field has been essential in our approach, since it provides a natural solution of the ordering ambiguities in the Hamiltonian, and gives us a field which ranges freely between $-\infty$ and $+\infty$. This field is defined by the integration of the relation $d r=\alpha d r_{*}$, and a problem arises as to the value of the integration constant. For instance, for the Schwarzschild black hole the general solution of this equation can be written as

$$
r_{*}=r+2 M \log \frac{r-2 M}{L_{0}}
$$

where $L_{0}$ is an arbitrary integration constant which is usually set equal to the horizon radius, $2 M$. Independently of $L_{0}, r_{*} \rightarrow-\infty$ as $r \rightarrow r_{+}$, and $r_{*} \simeq r$ if $r \rightarrow \infty$, so these boundary conditions do not help to fix $L_{0}$. All the steps leading to the Hamiltonian, eq. (32), can be repeated with $L_{0}$ generic, and we still obtain eq. (32) except that now $r$ as a function of $r_{*}$ is given by eq. (66) and thus depends on the arbitrary constant $L_{0}$. This introduces an ambiguity in our description. In the absence of a physical criterium for 

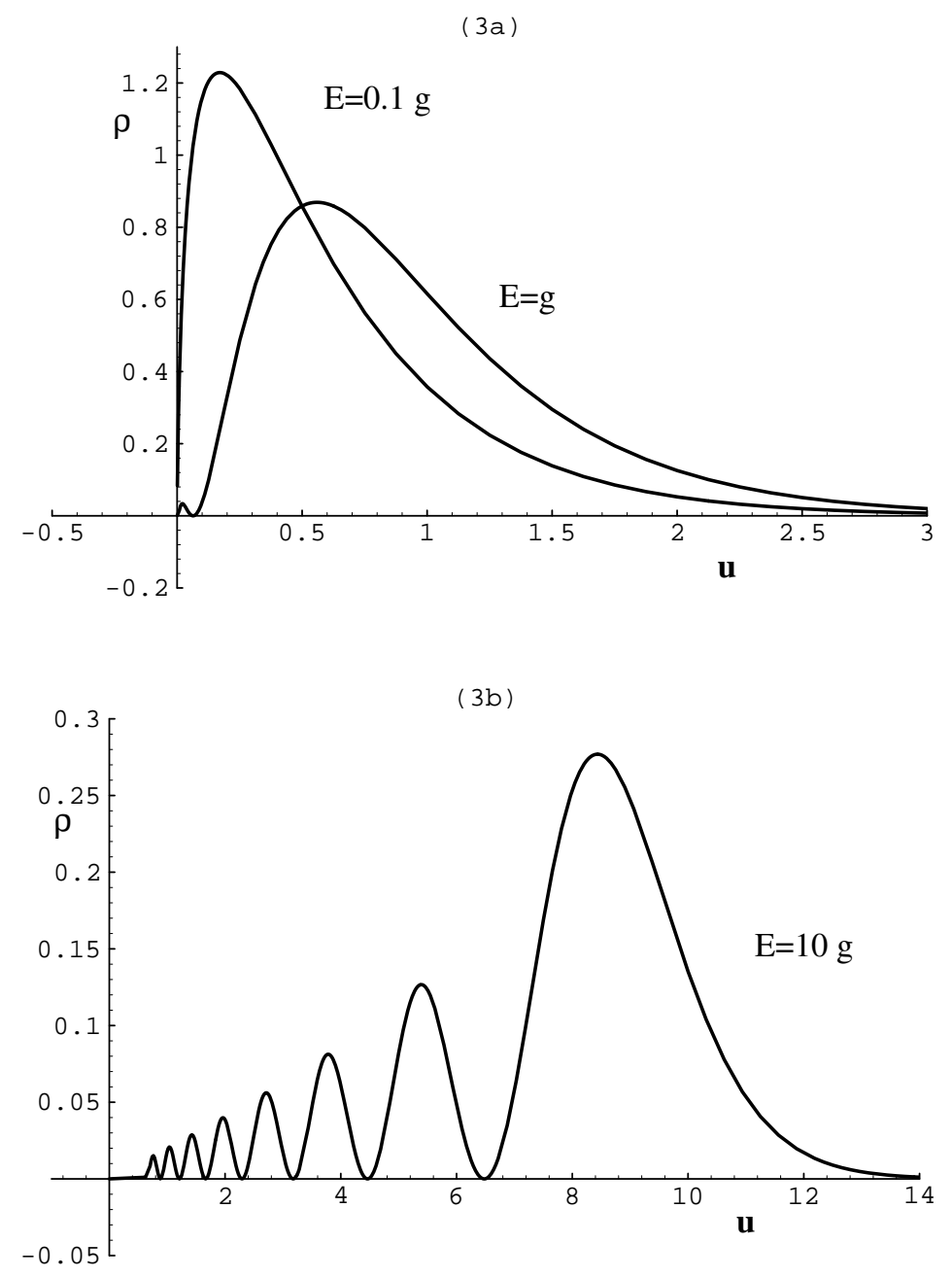

Figure 3: The probability distribution of the membrane, $\rho$, vs. $u=\mathcal{T}^{\prime} z$ for $E / g=0.1,1$ (fig. 3a) and for $E / g=10$ (fig. 3b). 
fixing this constant, we can proceed as follows. First, since the only length scale for a Schwarzschild black hole is given by the horizon radius, we can set $L_{0}=2 M l_{0}$, where $l_{0}$ is a numerical constant. We can now require that the constant $l_{0}$ gives only subleading effects. It is apparent from equation (66) that this happens if and only if $\log M$ is a parametrically large quantity, so that $2 M \log M$ is much larger than $2 M \log l_{0}$. Thus $r\left(r_{*}\right)$, and therefore the Hamiltonian $H$, do not depend on $l_{0}$ in a first approximation. Hence, our membrane approach gives unambiguous answers in the domain

$$
M \gg 1 \quad \text { (Schwarzschild) . }
$$

Before proceeding to the general Reissner-Nordstrom case, it is useful to derive again this condition in a way which is more suitable for generalizations. We use the variables $x=r /(2 M), x_{*}=r_{*} /(2 M), l_{0}=L_{0} /(2 M)$; then $x=$ $x\left(x_{*}, l_{0}\right)$ defined implicitly by

$$
x_{*}=x+\log \frac{x-1}{l_{0}} .
$$

Taking the partial derivative with respect to $l_{0}$ we get

$$
\frac{\partial x}{\partial l_{0}}=\frac{x-1}{x l_{0}}
$$

Consider the region $r \leq 2 M+$ const.; if we send $M \rightarrow \infty$ while keeping the constant arbitrarily large but fixed, then in this region $x \rightarrow 1$ and equation (69) shows that in this limit $x$ becomes independent of $l_{0}$. Thus, if $M \gg 1$, we have $\partial r / \partial l_{0} \ll 2 M$, which is the desired result since (when we restore conventional units) $2 G M$ is the only length scale in the problem.

Let us repeat this analysis for a non extremal Reissner-Nordstrom black hole; the relation between $r_{*}$ and $r$, with an appropriate definition of the integration constant $L_{0}$, is

$$
r_{*}=r+\frac{r_{+}^{2}}{r_{+}-r_{-}} \log \frac{r-r_{+}}{L_{0}}-\frac{r_{-}^{2}}{r_{+}-r_{-}} \log \frac{r-r_{-}}{L_{0}} .
$$

We define $x=r / r_{+}, l_{0}=L_{0} / r_{+}, \rho=r_{-} / r_{+}$. It is convenient to define also $\varepsilon, \delta$ from $x=1+\varepsilon, \rho=1-\delta$. Computing $\partial x / \partial l_{0}$ we find

$$
\frac{\partial x}{\partial l_{0}}=\frac{1}{l_{0}} \frac{(2-\delta) \varepsilon(\varepsilon+\delta)}{(1+\varepsilon)^{2}}
$$


In the Schwarzschild case we required $\partial x / \partial l_{0} \ll 1$ since this implies $\partial r / \partial l_{0} \ll$ $2 M$ and $2 M$ is the only scale in the problem. Here we also have a second scale, $\sqrt{M^{2}-Q^{2}}$. If we ask that $\partial r / \partial l_{0}$ should be small compared to any physical scale involved, we must require $\partial r / \partial l_{0} \ll \sqrt{M^{2}-Q^{2}}$. Then we get the condition

$$
\varepsilon(\varepsilon+\delta) \ll \delta .
$$

For black holes far from the extremal limit, $\delta \sim 1$, we recover the condition $\varepsilon \ll 1$, or $M \gg 1$. For near-extremal black holes we have $\delta \simeq 2 \sqrt{M^{2}-Q^{2}} / M$ and therefore $\delta$ is much smaller than $\varepsilon \sim$ const./M. The condition (72) then gives $\varepsilon^{2} \ll \delta$. In terms of $M, Q$ this means

$$
\sqrt{M^{2}-Q^{2}} \gg \frac{\text { const. }}{M} \text {. }
$$

Quite remarkably, this is the same result obtained in ref. [14] as the limit of validity of the thermodynamical description of black holes, in the case of zero angular momentum. be examined from the membrane point of view after the discussion of the energy spectrum of black holes in the next section. The region of validity of the membrane description is the area below the curve in fig. 4 , with the condition $M \gg 1$. Note that extremal black holes are excluded by this domain of validity, although, if $M$ is sufficiently large, we can get as close as we wish to the extremal limit.

A second source of worries for the consistency of our approach is due to the fact that we are studying the quantum theory obtained applying the minisuperspace approximation to the action, eq. (11) or eq. (2), but we are neglecting loop corrections to these actions which arise at the quantum level. However, it is easy to see what is the parameter governing these corrections. Restoring $\hbar$, the parameter of the loop expansion is $\hbar / \mathcal{T}^{\prime}$ and therefore the expansion is under control if $\mathcal{T}^{\prime}$ is sufficiently large. If one computes field theoretical corrections in the region outside the horizon, one encounters integrals over the three dimensional space which only extends to the region $r>r_{+}$, so that the relevant length scale is $r_{+}$. Since $\mathcal{T}^{\prime} \sim(\text { mass })^{3}$, the loop

\footnotetext{
${ }^{5}$ In the more general case of non-zero angular momentum, one finds [14 $Q^{2} \rightarrow Q^{2}+$ $\left(J^{2} / M^{2}\right)$ in eq. (73). It is clear that the same result would be obtained generalizing the membrane approach to $J \neq 0$, since the derivation only uses the definition of the tortoise coordinate.
} 


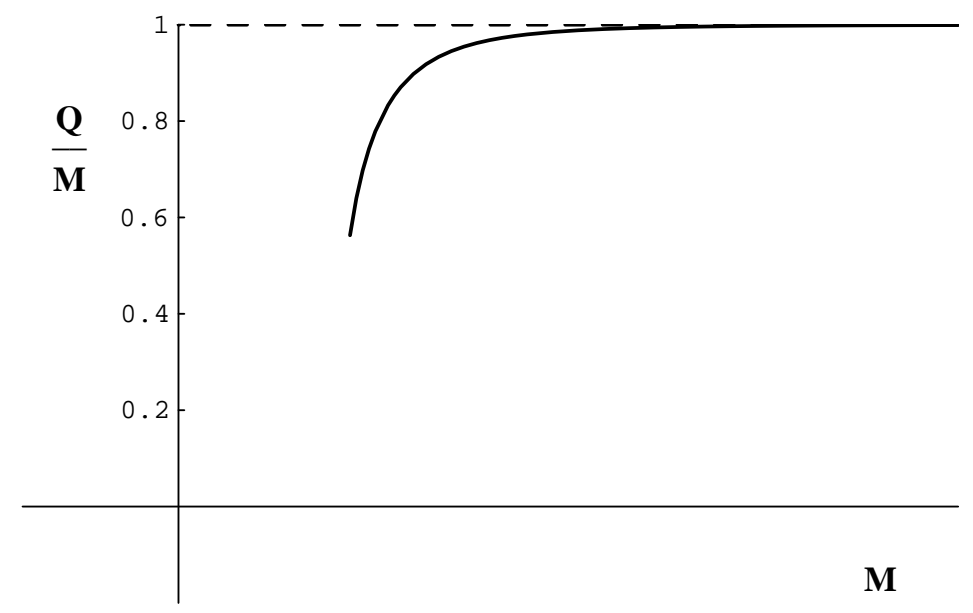

Figure 4: The domain of validity of the membrane description.

expansion is under control if (setting again $\hbar=1$ )

$$
\mathcal{T}^{\prime} r_{+}^{3} \gg 1
$$

This can be made more precise computing the effective static potential for the membrane. The computation is similar to the bosonic string case [30, 31]. However, because of the intrinsic non-linearity of the membrane action, it is necessary to find first a classical solution around which to expand the partition function. In ref. 24] the computation is performed considering a membrane embedded in a space-time with topology $S^{2} \times R^{D-2}$, where there are static solutions. Denoting by $R$ the radius of the sphere $S^{2}$, the effective potential for the closed bosonic membrane is, for large $R$, and in the large $D$ limit [24],

$$
V_{\text {eff }} \simeq \mathcal{T}^{\prime} R^{2}+\frac{(D-3)}{2} \frac{0.2651}{R} .
$$

Extrapolating the result to $D=4$, we see that the second term is small compared to the leading term if $\mathcal{T}^{\prime} R^{3} \gg 1$. This result is quite transparent physically. The situation is completely analogous to what happens when we try to describe the large distance effects in $Q C D$ in terms of a bosonic string, so that it is useful to keep this example in mind. The leading term is analogous to the linearly rising potential in the case of strings, $V_{\text {eff }} \sim \sigma R$, 
which gives rise to confinement in $Q C D$. The fact that for membranes $V_{\text {eff }} \sim$ $R^{2}$ while for strings it is linear in $R$ is dictated by dimensional arguments,

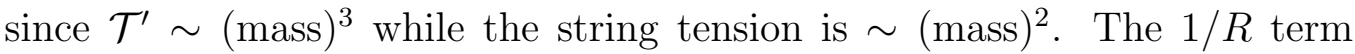
is generated by the transverse fluctuations and it is therefore proportional to the number of transverse degrees of freedom, $D-3$ for a membrane and $D-2$ for a string. It is also interesting to observe that, in the large $D$ limit, the effective potential due to the action (1D) and due to the action (8) are the same at first order in the $1 / D$ expansion [32].

It is clear that in the case of membranes in a Reissner-Nordstrom metric the role of $R$ is played by $r_{+}$. The leading term is the potential $V_{\mathrm{ph}}$ that we have previously defined as the square root of $V\left(r_{*}\right)$, eq. (35), and for large values of $r$ we have found $V_{\mathrm{ph}} \sim \mathcal{T}^{\prime} r^{2}$. The one-loop corrections are $\sim 1 / r$ and therefore they are under control if $\mathcal{T}^{\prime} r^{3} \gg 1$. Since $r \geq r_{+}$, condition (74) ensures that these corrections are small and that our approach is consistent.

For bosonic strings there is a critical distance, $\sigma R_{c}^{2} \sim(D-2)$, below which the effective potential becomes complex and the string picture breaks down [31]. This is quite clear physically, since at short distances the quark-antiquark dynamics is governed by asymptotic freedom rather then by confinement. Similarly for membranes [24] there is a critical distance $\mathcal{T}^{\prime} R_{c}^{3} \sim D-3$ below which the approach breaks down.

We have therefore found two conditions for the consistency of the membrane approach, eq. (73) and eq. (74). In the next section, after having discussed the energy level structure of black holes, we will be able to give a tentative estimate of the membrane tension $\mathcal{T}^{\prime}$. Then, we will find that these two conditions are actually one and the same.

\section{The energy spectrum of black holes}

In this section we study the level structure of black holes which is suggested by the membrane description. Some of our considerations in this section have only heuristic value, and the corresponding results should only be considered as an indication of how a black hole might look like at the quantum level.

Let us begin by considering the process in which a photon of energy, say, $E=1 \mathrm{MeV}$ falls into a Schwarzschild black hole of mass $M$. In the classical description, the Schwarzschild radius increases by an amount $\Delta r=2 G E$ which, for $E \sim 1 \mathrm{MeV}$, is $\Delta r \sim 10^{-22} L_{\mathrm{Pl}}$, where $L_{\mathrm{Pl}}$ is the Planck length. 
Now, it is quite obvious that such a result does not go through at the quantum level. Most probably, no meaning whatsoever can be attached in quantum gravity to such an exceedingly small distance, nor in general to distances smaller than $L_{\mathrm{Pl}}$. More specifically, in the case of the black hole horizon a Gedanken experiment [13] indicates that the measurement of the radius of the horizon is subject to a generalized uncertainty principle,

$$
\Delta x \gtrsim \frac{\hbar}{\Delta p}+\text { const. } G \Delta p,
$$

which implies the existence of a minimal measurable distance on the order of $L_{\mathrm{Pl}}$. A similar uncertainty principle was previously found in string theory [33, 34, 35, 36].

Because of this, it is more natural to assume that when a single particle with energy much smaller than the Planck mass is dropped into a black hole, the area of the horizon simply does not change. Or, in other words, that the area is quantized, and that the quantum of area is of order one in Planck units.

This argument, combined with the results of the previous section, leads us to suggest the following pattern for the level structure of black holes. Firstly, we have a "principal" series of levels, corresponding to the area quantization. We can describe them with a "principal quantum number" $n$, and at least for large $n$ we expect $A=A_{0} n$, where $A_{0}$ is the quantum of area, $A_{0} \sim L_{\mathrm{Pl}}^{2}$. Each level of the principal series is characterized by a well-defined value of $M$ and $Q$, and to each of these levels we can apply the membrane description developed in the previous section, inserting for $g_{\mu \nu}$ in the action, eq. (1), the Reissner-Nordstrom metric with the given values of $M, Q$. Thus, from each principal level starts a continuum (or more precisely a quasi-continuum, see below) of membrane levels, which are able to absorb, thermalize and reemit quanta with energy much smaller then the Planck mass.

In our approach, we can consistently discuss only levels in which the excitation energy of the membrane is sufficiently small, so that the quasicontinuum which starts at the level with principal quantum number $n$, i.e. at $E=E_{n}$, ends (much) before $E=E_{n+1}$. As discussed in sect. 3.1, the reason for this is that we have always neglected the back-reaction of the membrane on the metric. Of course, when the membrane absorbes an infalling particle and jumps to an excited level, the excitation energy $E$ gives 
a contribution to the metric. If the energy of the infalling particle is sufficiently small it is legitimate to neglect this contribution. The approximation however breaks down when the excitation energy becomes comparable with the spacing between principal levels.

This level structure is sketched in fig. 5. We will now discuss in turn these two type of levels.

\subsection{The principal levels}

The physical argument presented above suggests that the area is quantized and, at least for large $n$,

$$
A=A_{0} n,
$$

where $A_{0}$ is the area quantum. It is very interesting to note, in this connection, that the fact that in quantum gravity the area of physical surfaces is quantized has been suggested, from quite a different perspective, by Ashtekar, Rovelli and Smolin [37] and Rovelli [38 by making use of the loop representation [39]. The quantum of area in this approach is $A_{0}=\frac{1}{2} L_{\mathrm{Pl}}^{2}$, that is $A_{0}=1 / 2$ in Planck units. In the following, however, we will keep $A_{0}$ generical. For a Schwarzschild black hole, $A=16 \pi M^{2}$. Setting $M=E_{n}$, the principal levels are therefore given by

$$
E_{n}=\left(\frac{A_{0} n}{16 \pi}\right)^{1 / 2}
$$

and the spacing between two successive principal levels is, for large $n$,

$$
\Delta E_{n}=\frac{A_{0}}{32 \pi E_{n}}
$$

For a Reissner-Nordstrom black hole $A=4 \pi r_{+}^{2}$ and one immediately finds the more general level formula

$$
E_{n}=\sqrt{\frac{A_{0} n}{16 \pi}}\left(1+\frac{4 \pi Q^{2}}{A_{0} n}\right) .
$$

If we set $A_{0}=8 \pi$ we recover a level formula which was proposed already in 1974 by Bekenstein [4], who argued that classically the irreducible mass squared of a black hole behaves as an adiabatic invariant, and then used the 


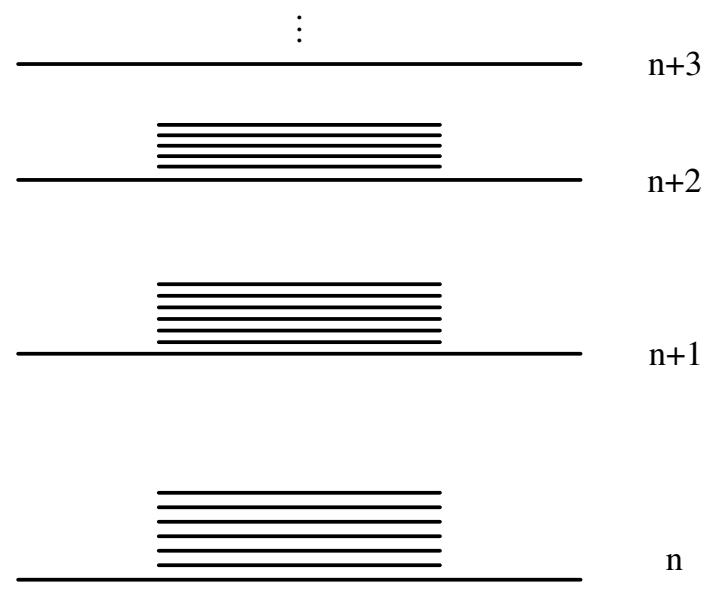

Figure 5: The level structure of black holes. 
Bohr-Sommerfeld quantization condition. The spacing between successive levels is

$$
\begin{gathered}
\Delta E_{n}=\frac{A_{0}}{8 \pi} \kappa_{n}, \\
\kappa_{n}=\frac{\sqrt{E_{n}^{2}-Q^{2}}}{\left(E_{n}+\sqrt{E_{n}^{2}-Q^{2}}\right)^{2}} .
\end{gathered}
$$

The choice $A_{0}=8 \pi$ has therefore an intuitive justification in the membrane approach: we have found in sect. 3 that at the first quantized level the dynamics is governed by a "physical" potential corresponding to exchange of a Yukawa particle of mass $\kappa_{n}$. This suggests that, if one develops a second quantized formalism, the successive levels of the principal series will correspond to levels which differ by one unit in the occupation number of this Yukawa particle, and then $\Delta E_{n}=\kappa_{n}$.

\subsection{The membrane levels}

In sect. 3 we have found levels corresponding to excitations of the membrane. Within our model we cannot ascertain whether these levels really form a continuum, as we found in the above analysis, or they are discrete, although very finely spaced. The continuum of levels is due to the fact that the potential $V\left(r_{*}\right)$ goes to zero as we approach the horizon. From the point of view of a fiducial observer, however, the Hawking temperature diverges on the horizon, because of the infinite red-shift factor between the horizon and infinity. Thus, if $r<r_{+}+\delta$, with $\delta$ on the order of a few Planck lengths, we are entering a region of superplanckian temperatures, where new physics, about which we are almost totally ignorant, comes into play. This is in fact one of the motiviations for constructing the stretched horizon [6, 5]. Thus, the form of the potential $V\left(r_{*}\right)$ at a few Planck length from the nominal horizon is not under control; or, in other words, our model of a black hole horizon as an infinitely thin membrane cannot be consistently used down to distances $\sim L_{\mathrm{Pl}}$ from the nominal horizon.

The question of whether these levels form a continuum or are discrete is a very important one and it is strictly related to the information loss paradox. If the levels actually form a continuum, the number of states in a finite volume $r_{+} \leq r \leq r_{\max }$ is divergent, no matter how close $r_{\max }$ is to $r_{+}$. Correspondingly, the entropy associated to the horizon also diverges. 
This divergence is the same which was found by 't Hooft [2] computing the entropy of a scalar field in the vicinity of the horizon. It can be regularized requiring that the scalar field vanishes within some fixed distance from the horizon. 't Hooft called this regulator the "brick wall" model. As stressed in ref. [2] and more recently in ref. [40], the information loss paradox can be traced back to this divergence.

Since without a detailed knowledge of physics at length scales on the order of the Planck length we cannot decide whether the levels really form a continuum, the information loss problem actually belongs to the domain of a full quantum theory of gravity, as claimed in ref. [9]. However, even if strictly speaking the membrane approach, as we have presented it, cannot give an unambiguous answer to the information loss problem, it is clear that it certainly does not support the claim of Hawking that unitarity is violated in black hole evaporation [1], since we have at our disposal a much more simple and economical possibility, namely that because of the effect of the stretched horizon the energy levels of the membrane become discrete. In the following, we will therefore assume that this is indeed the case.

At least at a qualitative level, we can reproduce the features of the stretched horizon imposing by hand a cutoff at $r=r_{+}+\delta$ and requiring $\psi\left(r_{*}\right)$ to be zero at the cutoff, as in ref. [2]. In other words, we modify the potential near the horizon, so that it has the form depicted in fig. 6. Thus, the energy levels become discrete, although finely spaced.

However, the levels shown in fig. 6 only represent radial excitations of the black hole, because our minisuperspace approximation has frozen all degrees of freedom except for the radius of the membrane. It is quite obvious that these radial excitations are not relevant at all for the description of the interaction between black holes and infalling particles. This is also indicated by the fact that the spacing between these levels is even larger than the spacing between the corresponding principal levels; in fact, approximating $V\left(r_{*}\right)$ with a square potential well and remembering that the role of the eigenvalue is played by $E^{2}$ (see eq. (34)), we immediately find $E_{n k}=E_{n}+\left(2 \pi \kappa_{n} k / \log E_{n}\right)^{1 / 2}$, where $k$ is an integer enumerating the spherical membrane levels, and for large $n$ this gives a spacing larger than $\Delta E_{n}$.

On the other hand, when the black hole absorbes a localized particle it does not make a transition between levels with spherical symmetry. Rather, classically a small bump is formed on the horizon (and subsequently reab- 


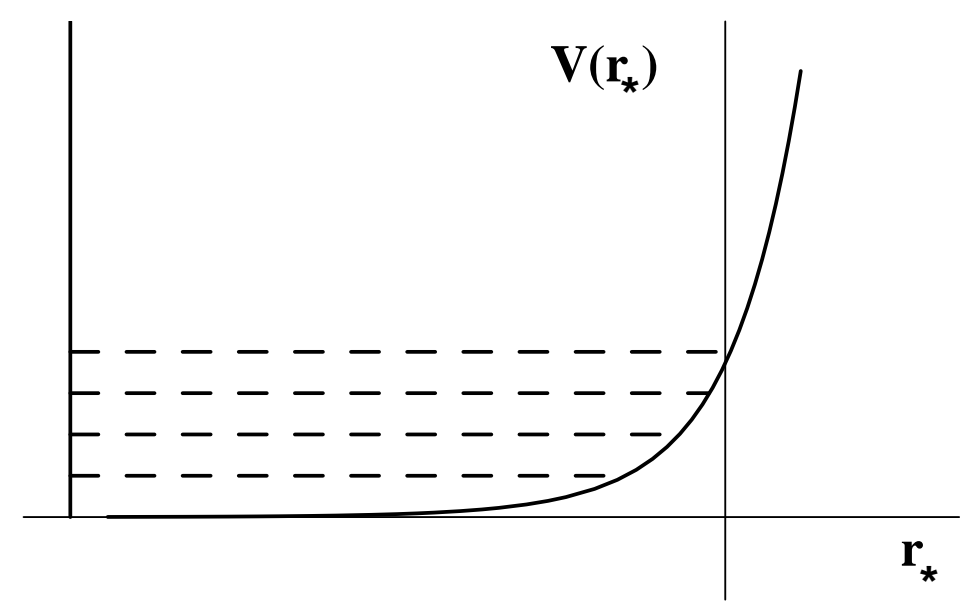

Figure 6: The potential modified with an infinite wall at the stretched horizon. The energy levels (dashed lines) become discrete. 
sorbed) close to the impact point. In the quantum description, this corresponds to the existence of a very large number of levels, describing states with slight deviations from spherical symmetry, which cannot be studied within the minisuperspace approximation, and which are much more finely spaced than the radial excitations. Since the bump can form on any place on the surface, the degeneration of these levels grows with the area of the horizon.

The fact that the spectrum is not continuous anymore implies that a black hole cannot absorb arbitrarily small amounts of energy. At first sight this might be surprising, since if a particle moves radially toward the center of the black hole, we naively expect that it will always be absorbed. However, this naive expectation fails if the De Broglie wavelength of the particle is sufficiently large compared to the horizon radius, since in this case the amplitude of localizing the particle close to the horizon is small. Thus, we expect that the separation between the membrane levels in a state with principal quantum number $n$ will be proportional to an appropriate inverse power of $E_{n}$.

Another important point concerning the membrane levels is the existence of an upper cutoff on the excitation energy of the membrane. In sect. 3.1 we derived a bound for the excitation energy, eq. (40), from the condition that the variation of the area of the horizon should be small compared to a Planck unit of area. On the other hand, in this section we have proposed a level formula which gives for the spacing between principal levels $\Delta E_{n}=\kappa_{n}$ (setting $A_{0}=8 \pi$ ). It is quite natural to assume that the maximum excitation energy of the membrane allowed by eq. (40) is on the order of $\Delta E_{n}$; in fact, when the bound (40) is saturated the membrane wave function is not yet suppressed at values of $r$ corresponding to an area of the horizon one Planck unit larger than the area of the nominal horizon $4 \pi r_{+}^{2}$. On the other hand, $\Delta E_{n}$ is just the spacing between levels which differ by a Planck unit of area. This allows us to give a tentative estimate of the value of the membrane tension. Note that, since the membrane action, eq. (2), describe the excitations over a state with $M, Q$ given, also $\mathcal{T}^{\prime}$, as well as the metric $g_{\mu \nu}$, can in principle depend on $M, Q$. Then, from eq. (40) and from $\Delta E_{n}=\kappa_{n}$ we obtain

$$
\mathcal{T}^{\prime 2} \sim \frac{\kappa_{n}}{r_{+}^{3}}
$$

where here $r_{+}=E_{n}+\sqrt{E_{n}^{2}-Q^{2}}$. Assuming the correctness of this estimate, 
the limit of validity of the membrane description, given in eq. $(\sqrt{73})$, takes the form

$$
\mathcal{T}^{\prime} r_{+}^{3} \gg 1
$$

which is just the condition obtained in sect. (3.3) requiring that the loop corrections to the membrane action should be small. Thus, the two consistency conditions obtained in sect. (3.3) are one and the same, and are in turn equivalent to the limits of validity of the statistical approach to black holes, found in ref. [14]; this is quite satisfying and indicates that our effective description has a good degree of inner consistency.

\section{Radiation from an accelerated charge and the membrane approach}

In this paper, we have mostly studied the energy level structure of quantum black holes. We have not discussed how to compute transition amplitudes between these levels. However, an interesting observation can be made in the case of Rindler space. Let us consider, following ref. [41], a massless scalar field coupled to a source in flat Minkowski space,

$$
L=-\frac{1}{2} \partial_{\mu} \phi \partial^{\mu} \phi+\rho \phi,
$$

where the source $\rho$ is uniformly accelerating. We denote by $(T, x, y, Z)$ the flat Minkowski coordinates and define the coordinates $z, t$ as in eqs. (20). Let us define the usual Rindler coordinate $\xi$ from $g z=\exp (g \xi)$. ( $\xi$ coincides with $z_{*}$ if we set $z_{0}=1 / g$ in eq. (51)).

The equation of motion of a uniformly accelerating particle is $\xi=$ const. In ref. 41] (see also [42]) it is considered the case

$$
\rho=\sqrt{2} q \cos (E t) \delta(\xi) \delta(x) \delta(y)
$$

corresponding to a uniformly accelerating charge in Minkowski space. Furthermore, the value of the charge is oscillating with frequency $E$. In refs. 42, 41] the factor $\cos (E t)$ is inserted only as a regulator, and the limit $E \rightarrow 0$ is taken at the end of the computation. However, the case $E \neq 0$ is also interesting in its own right. The vacuum state in Minkowski space corresponds, in the accelerating frame, to a thermal bath with temperature $T=g /(2 \pi)$. 
Thus, the comoving observer sees a charge interacting with a thermal bath, and will therefore observe spontaneous and stimulated emission, as well as absorption. The corresponding rates have been computed in ref. [41]. The total emission rate of quanta with transverse components of the momentum $k_{x}, k_{y}$ is found to be

$$
d W^{\mathrm{em}}\left(k_{x}, k_{y}\right)=\frac{q^{2}}{4 \pi^{3} g} \sinh \left(\frac{\pi E}{g}\right)\left[\frac{1}{e^{2 \pi E / g}-1}+1\right]\left|K_{i \frac{E}{g}}\left(\frac{k_{\perp}}{g}\right)\right|^{2},
$$

where $k_{\perp}^{2}=k_{x}^{2}+k_{y}^{2}$ and $K$ is the MacDonald function. The first term in the square bracket gives the induced emission and shows that the particle sees a thermal bath with temperature $T=g /(2 \pi)$. The second term gives the spontaneous emission rate.

The above result has a rather suggestive interpretation in the membrane approach. From the results of section 3.2, the wave function of the membrane corresponding to the excitation energy $E$ is

$$
\psi_{E}(u)=\left(\frac{\sqrt{2} \sinh (\pi E / g)}{\pi g}\right)^{1 / 2} K_{i \frac{E}{g}}(u),
$$

where, in terms of $\xi, u=\left(\mathcal{T}^{\prime} / g\right) \exp (g \xi)$ and we use the normalization given in eq. (59). We see a striking similarity between the spontaneous emission rate computed in ref. 41 and the quantity

$$
\begin{aligned}
d W & =\frac{1}{8 \pi^{2}}\left|\int d^{3} x \psi_{E}^{*} q \delta(x) \delta(y) \delta(\xi) \psi_{E}\right|^{2}= \\
& =\frac{q^{2}}{4 \pi^{3} g} \sinh \left(\frac{\pi E}{g}\right)\left|K_{i \frac{E}{g}}\left(\frac{\mathcal{T}^{\prime}}{g}\right)\right|^{2} .
\end{aligned}
$$

The integral has a natural interpretation as a matrix element of the charge operator computed with the membrane wave function with excitation energy $E$. The similarity is only approximative, since the argument of the MacDonald function in eq. (89), after performing the integral with the help of the Dirac delta, is $\mathcal{T}^{\prime} / g$, while in eq. (87) it is $k_{\perp} / g$. (Note that in the Rindler case $\mathcal{T}^{\prime}$ has dimensions of mass, since $\mathcal{T}^{\prime}=\mathcal{T}\left(\int d x d y\right)$.) It is clear that such a dependence cannot be obtained in our minisuperspace approximation, which has frozen all transverse degrees of freedom. Still, the appearence in both equations of quite non trivial factors as the MacDonald function of order $i E / g$ 
squared, or the factor $\sinh (\pi E / g)$ is rather suggestive, and it is difficult to consider it as purely accidentental.

Of course, our attempt to describe quantum black holes in terms of membranes is still in a preliminary stage and, as mentioned in the Introduction, we are still trying to understand what are "the rules of the game". The above result might be taken as an indication of the fact that it may be possible to describe quantitatively the Hawking radiation in terms of transitions between membrane levels.

\section{Conclusions}

The overall picture of black holes that emerges from our analysis is quite in agreement with that discussed, e.g., in ref. [3]. There is a large number of low lying levels which absorb and thermalize the energy of infalling particles. The energy is therefore emitted in a way which, from a practical point of view, has lost any memory of the initial state. However, this is not different from what happens in any ordinary macroscopic body, or in the liquid-droplet model of the nucleus and at the fundamental level the evolution is unitary. The membrane levels are just the "quantum hair" which have been looked

for (see e.g. ref. [43]) in order to solve the information loss problem raised by Hawking without abandoning unitarity. For a given value of mass and charge, we have found that the state of a non rotating black hole is not uniquely determined, contrarily to what happens in classical general relativity (no hair theorem), but rather there exists a quasi-continuum of membrane levels. These microstates are missed in the classical description, in which a state is only described with the "macroscopic" variables $M, Q$ and therefore reappear in the form of entropy.

In our picture, the Hawking radiation is just the spontaneous decay of the levels that we have found. The apparently thermal character of the radiation stems from the existence of a large number of levels, and the order of magnitude of the temperature is fixed by the spacing between successive principal levels, i.e. $T \sim \kappa$. The precise value of the coefficient $(1 /(2 \pi)$ from Hawking calculation) cannot be computed within our minisuperspace approximation, as well as we cannot compute the proportionality constant between the entropy and the area. The radiation, however, only appears thermal as far as the energy spectrum is concerned, but there are correlations 
between earlier and later radiation.

Our approximations are not justified when the mass of the black hole becomes of the order of the Planck mass. Thus, we cannot follow the evaporation till the endpoint. At some point of the evaporation process we reach a value of $M, Q$ for which the membrane approach breaks down. This happens at the same point when the thermodynamical description of black holes also breaks down. This is understandable, since when the membrane approach

is valid we have a large number of low lying levels for each principal level and a statistical description is therefore appropriate. On the other side of the dividing line in fig. 4 we rather expect that a black hole resembles much more an elementary particle, or a normal quantum sistem with few degrees of freedom, rather than a macroscopic extended object.

Acknowledgments. I thank Enore Guadagnini, Michele Mintchev and Gabriele Veneziano for useful discussions.

\section{References}

[1] S.W. Hawking, Phys. Rev. D14 (1976) 2460.

[2] G. 't Hooft, Nucl. Phys. B256 (1985) 727.

[3] C.F.E. Holzhey and F. Wilczek, Nucl. Phys. B380 (1992) 447.

[4] J. Bekenstein, Lett. Nuovo Cimento, 11 (1974) 467.

[5] L. Susskind, L. Thorlacius and J. Uglum, Phys. Rev. D48 (1993) 3743.

[6] K.S. Thorne, R.H. Price and D.A. Macdonald, "Black Holes: The Membrane Paradigm", Yale Univ. Press, 1986, and references therein.

[7] G. 't Hooft, Nucl. Phys. B335 (1990) 138; Physica Scripta T36 (1991) 247.

[8] L. Susskind, "String Theory and the Principle of Black Hole Complementarity", preprint SU-ITP-93-18, July 1993, hep-th/9307168. 
[9] L. Susskind and L. Thorlacius, "Gedanken Experiments Involving Black Holes", preprint SU-ITP-93-19, August 1993, hep-th/9308100.

[10] L. Susskind, "Strings, Black Holes and Lorentz Contraction", preprint SU-ITP-93-21, August 1993, hep-th/9308139.

[11] C.R. Stephens, G. 't Hooft and B.F. Whiting, "Black hole evaporation without information loss", preprint THU-93/20, gr-qc/9310006.

[12] K. Schoutens, H. Verlinde and E. Verlinde, "Black Hole Evaporation and Quantum Gravity", preprint CERN-TH.7142/94, hep-th/9401081.

[13] M. Maggiore, Phys. Lett. B304 (1993) 65; "Black Hole Complementarity and the Physical Origin of the Stretched Horizon", preprint IFUPTH 44/93, Oct. 1993, Phys. Rev. D, to appear.

[14] J. Preskill, P. Schwarz, A. Shapere, S. Trivedi and F. Wilczek, Mod. Phys. Lett. A6 (1991) 2353.

[15] U. Marquard and M. Scholl, Phys. Lett. B227 (1989) 227;

I. Bars, Nucl. Phys. B343 (1990) 398.

[16] P.A.M. Dirac, Proc. Roy. Soc. London A 268 (1962) 57.

[17] P.A. Collins and R.W. Tucker, Nucl. Phys. B112 (1976) 150.

[18] P. Gnadig, Z. Kunszt, P. Hasenfratz and J. Kuti, Ann. Phys. 116 (1978) 380.

[19] A. Aurilia, G. Denardo, F. Legovini and E. Spallucci, Nucl. Phys. B252 (1985) 523;

A. Aurilia, R.S. Kissack, R. Mann and E. Spallucci, Phys. Rev. D35 (1987) 2961.

[20] A. Aurilia and E. Spallucci, Phys. Lett. B251 (1990) 39.

[21] J. Hughes, J. Liu and J. Polchinski, Phys. Lett. B180 (1986) 370;

E. Bergshoeff, E. Sezgin and P.K. Townsend, Phys. Lett. B189 (1987) 75 . 
[22] M.J. Duff, T. Inami, C.N. Pope, E. Sezgin and K.S. Stelle, Nucl. Phys. B297 (1988) 515.

[23] P.S. Howe and R.W. Tucker, J. Phys. A 10 (1977) L155.

[24] E.G. Floratos, Phys. Lett. B220 (1989) 61;

E.G. Floratos and G.K. Leontaris, Phys. Lett. B220 (1989) 65.

[25] A. Sugamoto, Nucl. Phys. B215 (1983) 381.

[26] K. Kikkawa and M. Yamasaki, Prog. Theor. Phys. 76 (1986) 1379.

[27] D.H. Hartley, M. Önder and R.W. Tucker, Class. Quantum Grav. 6 (1989) 1301.

[28] C. Ho and Y. Hosotani, Phys. Rev. Lett. 60 (1988) 885.

[29] E. D'Hoker and R. Jackiw, Phys. Rev. D26 (1982) 3517, appendix B.

[30] M. Lüscher, K. Symanzik and P. Weisz, Nucl. Phys. B173 (1980) 365.

[31] O. Alvarez, Phys. Rev. D24 (1981) 440;

J.F. Arvis, Phys. Lett. B127 (1983) 106.

[32] S.D. Odintsov and D.L. Wiltshire, Class. Quantum Grav. 7 (1990) 1499.

[33] G. Veneziano, Europhys. Lett. 2 (1986) 199; Proc. of Texas Superstring Workshop (1989).

D. Gross, Proc. of ICHEP, Munich (1988).

[34] D. Amati, M. Ciafaloni and G. Veneziano, Phys. Lett. B197 (1987) 81, B216 (1989) 41; Int. J. Mod. Phys. A3 (1988) 1615; Nucl. Phys. B347 (1990) 530.

[35] D.J. Gross and P.F. Mende, Phys. Lett. B197 (1987) 129; Nucl. Phys. B303 (1988) 407.

[36] K. Konishi, G. Paffuti and P. Provero, Phys. Lett. B234 (1990) 276;

R. Guida, K. Konishi and P. Provero, Mod. Phys. Lett. A6 (1991) 1487. 
[37] A. Ashtekar, C. Rovelli and L. Smolin, Phys. Rev. Lett. 69 (1992) 237.

[38] C. Rovelli, Nucl. Phys. B405 (1993) 797.

[39] C. Rovelli and L. Smolin, Phys. Rev. Lett. 61 (1988) 1155;

A. Ashtekar, "Non-perturbative canonical gravity", World Scientific, Singapore 1991, and references therein.

[40] L. Susskind, "Some speculations about black hole entropy in string theory", preprint RU-93-44, hep-th/9309145.

[41] H. Ren and E.J. Weinberg, "Radiation from a moving Scalar Source", preprint CU-TP-592, hep-th/9312038.

[42] A. Higuchi, G.E.A. Matsas and D. Sudarsky, Phys. Rev. D46 (1992) 3450 .

[43] S. Coleman, J. Preskill and F. Wilczek, Nucl. Phys. B378 (1992) 175. 